\title{
Efectividad del Colutorio de Manzanilla Comparado con Placebo y Clorhexidina en Pacientes con Gingivitis entre 19 y 25 Años: Ensayo Clínico Controlado
}

\author{
Effectiveness of Chamomile Mouthwash Compared with Placebo and Chlorhexidine in \\ Patients with Gingivitis Aged 19 to 25 Years: Controlled Clinical Trial \\ María Jesús Gaete Forno* \& Patricio Oliva Mella*
}

GAETE, F. M. J. \& OLIVA, M. P. Efectividad del colutorio de manzanilla comparado con placebo y clorhexidina en pacientes con gingivitis entre 19 y 25 años: ensayo clínico controlado. Int. J. Odontostomat., 6(2):151-156, 2012.

RESUMEN: La gingivitis es una enfermedad periodontal de alta prevalencia en la población. El control mecánico de la placa bacteriana y el uso de un agente químico ayudan a su tratamiento y prevención. El objetivo de esta investigación fue determinar la efectividad del colutorio de Manzanilla comparado con Placebo y Clorhexidina (0,12\%) en la reducción de la inflamación gingival en pacientes con gingivitis entre 19 y 25 años de edad. Se realizó un ensayo clínico, mediante muestreo no probabilístico participaron 30 sujetos que fueron randomizados en tres grupos asignando a cado uno de los tratamientos: Manzanilla, Clorhexidina $(0,12 \%)$ y Placebo. Se controló a la 7 y 15 días del uso del colutorio. Se evaluó el índice gingival, índice de placa y el índice hemorrágico. Se realizó análisis estadístico donde se mide la normalidad mediante Shapiro-Wilk, y ANOVA para medidas repetidas para la evaluación de los asociados. Los datos obtenidos muestran diferencias significativas en el análisis estadístico paramétrico y no paramétrico. EL colutorio de Manzanilla es efectivo en la reducción de la inflamación comparada con Placebo y Clorhexidina.

PALABRAS CLAVE: colutorio, manzanilla, clorhexidina, inflamación gingival.

\section{INTRODUCCIÓN}

La gingivitis es una enfermedad dental de alta prevalencia en la población mundial, asociada principalmente a la placa bacteriana. Estudios estiman que un tercio de la población de los países desarrollados elimina la placa bacteriana de forma mecánica (Mullally et al., 1995). Los medios mecánicos requieren mucho tiempo y las personas carecen de motivación para realizarla (Ozaki et al., 2006) por lo cual los agentes químicos son necesarios y ayudan al control bacteriológico en dicha patología (Mandel, 1998). El control mecánico de la placa bacteriana comúnmente se realiza a través del cepillado y ocasionalmente con el uso de la seda dental, procedimientos que son insuficientes, ya que no se eliminan las mayorías de las bacterias presentes en el medio oral (Bernimoulin, 2003). La utilización de productos naturales para el control de la placa bacteriana permite su uso frecuente con un mínimo de contraindicaciones. La Matricaria recutita Linn es una planta nativa de Europa y Asia occidental (Mckay \& Blumberg, 2006) conocida como Manzanilla. La manzanilla ha sido utilizada durante siglos como un antiinflamatorio, además posee propiedades como antioxidante, astringente (Weiss, 1998), analgésico, antiespmadódico y propiedades sedativas (Sard Lahijani et al., 2006).

La manzanilla no presenta efectos secundarios ni contraindicaciones, como ocurre con la clorhexidina que produce tinciones dentales después de 15 días de uso prolongado (Calsina-Gomis \& Serrano-Granger, 2005), hipertrofia reversible de la mucosa cambios en el gusto y aumento de los depósitos supragingivales calcificados (Torres et al., 2000). Es por esto que se ha buscado agentes naturales que tengan una óptima acción antimicrobiana con bajos efectos secundarios.

Los principales componentes del aceite de manzanilla son terpenos, a- bisabolol y su óxido azuleno

`Facultad de Odontología, Universidad del Desarrollo, Concepción, Chile. 
incluyendo camazuleno y derivados del acetileno (Srivastava et al., 2010). La propiedad antiinflamatoria de la manzanilla se debe al mecanismo de acción de inhibición de la producción de PGE2 que suprime la expresión del gen COX-2 y la inhibición directa de la actividad de la enzima COX-2 (Srivastava et al.). Sus características han sido probadas in vitro, in vivo y en procedimientos clínicos (Mckay \& Blumberg). La infusión (té) de manzanilla que ha sido utilizada en las enfermedades bucodentales relacionadas con procesos inflamatorios y / o infecciosas (Paixão et al., 2002). Se ha usado también en cremas, geles, colutorios y dentífricos (George et al., 2009).

Estudios in vitro han mostrado que tinturas de Matricaria camonilla poseen efectos sobre ciertos microorganismos formadores de biofilm comparable al de la clorhexidina (Pereira et al., 2006). Además se ha evidenciado que existe una reducción en el índice de placa e índice gingival con el uso dentífricos que tienen una base de extracto de manzanilla (Saxer et al., 1995).

Esta investigación tiene como objetivo determinar la efectividad del colutorio de Manzanilla comparado con Placebo y Clorhexidina en la reducción de la inflamación gingival en pacientes con gingivitis entre 19-25 años.

\section{MATERIAL Y MÉTODO}

Se realizó ensayo clínico randomizado, en que se comparan grupos paralelos, fase III, cuyo objetivo principal es determinar la efectividad del colutorio de Manzanilla comparado con Placebo y Clorhexidina $(0,12 \%)$ en la reducción de la inflamación gingival en pacientes con gingivitis.

Los participantes fueron identificados desde la Clínica Odontológica de la Universidad del Desarrollo, reclutando 30 sujetos, 13 hombres $(43,3 \%)$ y 17 mujeres $(56,7 \%)$ entre 18 y 25 años $(x=22,7)$. El tipo de muestreo fue no probabilístico por conveniencia. La muestra resultante del reclutamiento se randomizó por bloques por un asignador externo en tres grupos, en relación 1:1:1, con doble ciego, donde el ciego fueron los pacientes y el asignador.

El primero grupo (tratamiento) utilizó Colutorio de extracto de Matricaria recutita I, tipo Manzanilla Primavera Puelche, $(0,8 \%$ de alcohol, $10 \%$ de glicerina y
0,8\% de extracto de Matricaria recutita I, el segundo grupo estándar utilizó Clorhexidina $0,12 \%$ y el tercer grupo placebo (agua destilada, ácido ascórbico 0,02 mg y tinción verde menta.

Los criterios de inclusión fueron: a) profundidad de saco $\leq 3 \mathrm{~mm}$, b) sin pérdida de inserción, c) índice gingival Loe y Sillness $\geq 0,5$, d) mínimo 20 dientes, f) no haber estado en terapia periodontal en menos de un año y no estar durante el estudio y tener buena salud en general.

Los criterios exclusión fueron: a) estar embarazada, estar bajo terapia antibiótica los últimos 6 meses, b) sujetos a aparato removible y/o aparato de ortodoncia, c) presencia patología en la mucosa.

Los pacientes recibieron un consentimiento informado explicando el propósito del estudio y el procedimiento, debía ser comprendido y firmado. Esto de acuerdo al Comité de Ética de la Facultad de Odontología de la Universidad del Desarrollo. Todos los procedimientos realizados fueron de acuerdo a las normas de Helsinki, 2005.

Para la recolección de datos se utilizó una ficha clínica que incluyó los datos del paciente, un cuestionario para determinar si cumplían con los criterios de inclusión y exclusión, Índice Gingival y de Placa de Loe y Sillness y el Índice Hemorrágico. Se validó a través de un Juicio de Expertos a través del coeficiente Kappa.

No se realizó una profilaxis previa y tampoco se intentó modificar los hábitos de higiene bucal. Para el examen se evaluó tres parámetros: Índice Gingival (Pourabbas et al., 2005), Índice de placa e Índice Hemorrágico. Las mediciones del estudio fueron realizadas sólo por el investigador principal. Existe calibración del investigador que evalúa la remisión gingival.

Para el examen periodontal se utilizó: bandeja de examen, espejo y Sonda Carolina del Norte (Hufriedy). Luego se les entregó el colutorio asignado según la randomización junto con las indicaciones de uso. Esta frecuencia de uso clínico de la Manzanilla fue determinada en un estudio experimental (Cárcamo et al., 2008). Se realizaron controles a los 7 y 15 días.

Con los datos obtenidos se realizó un análisis descriptivo gráfico. El criterio de significancia estadística se estableció en $p<0,05$. Se determinaron los valores de normalidad utilizando el Test de ShapiroWilk. Se compararon las variables en los tres tiempos 


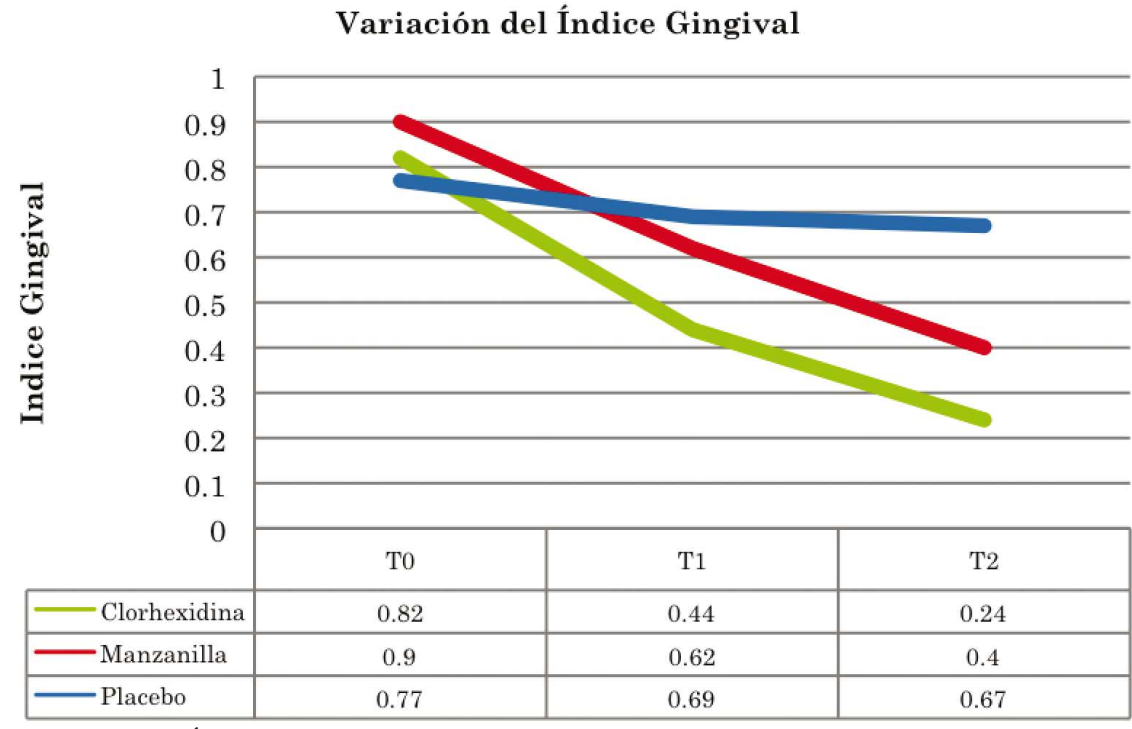

Variación del Índice Gingival de acuerdo al tiempo de exposición del tratamiento.

Variación del Índice de Placa

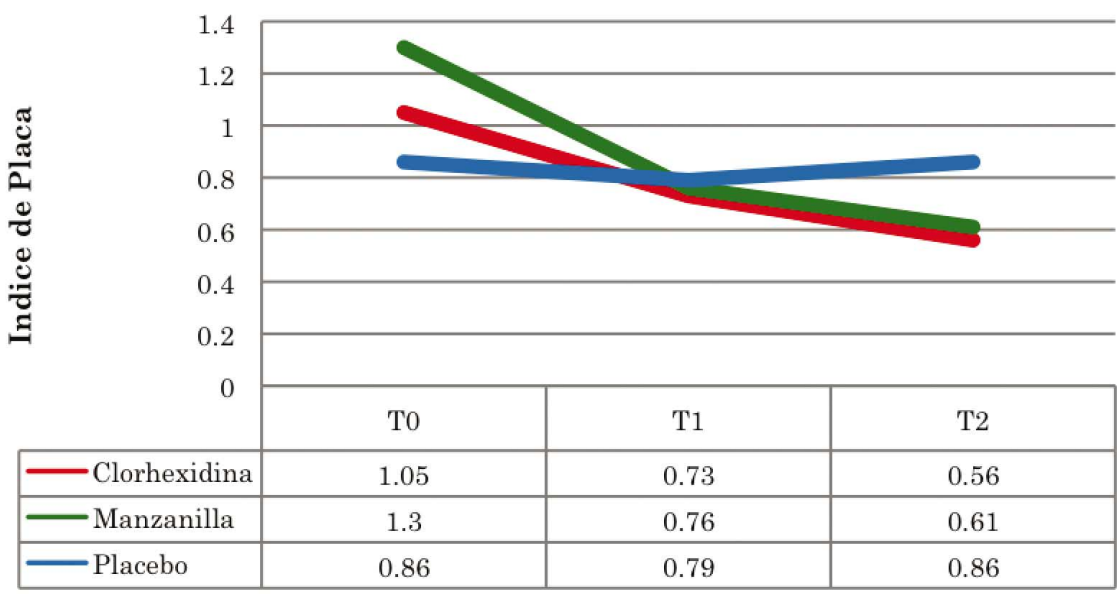

Variación del Índice de Placa de acuerdo al tiempo de exposición del tratamiento.

\section{Variación Índice de Hemorrágico}

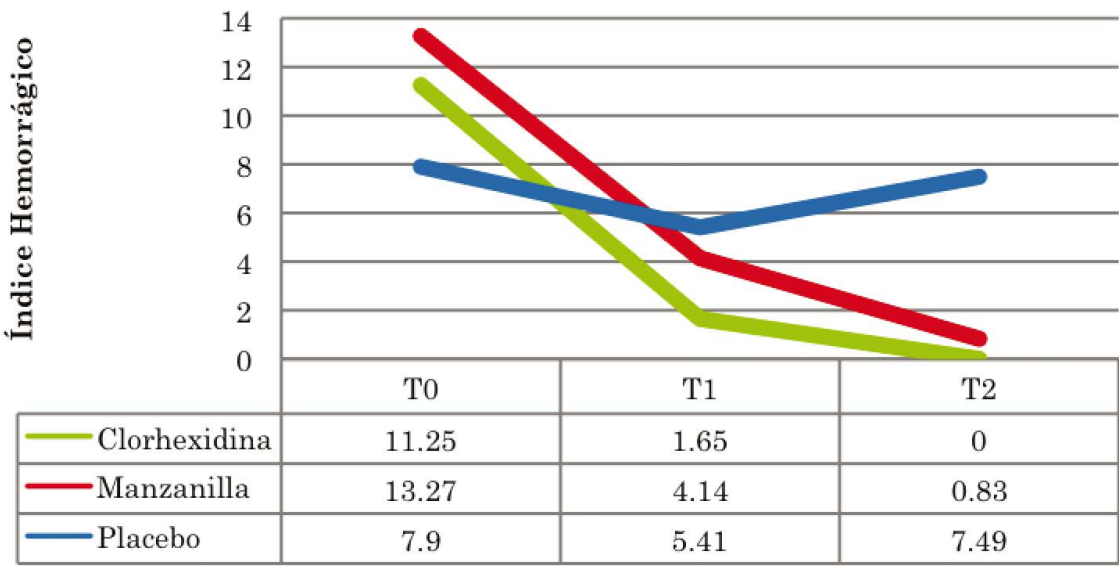

Variación del Índice Hemorrágico de acuerdo al tiempo de exposición del tratamiento. para cada tratamiento, como son mediciones dependientes en el tiempo se utiliza ANOVA de medidas repetidas para los valores paramétricos y cuando los valores son paramétricos se ocupó la Dócima de Friedman. Se compararon en cada tiempo la efectividad del tratamiento para cada colutorio.

\section{RESULTADOS}

Se evaluó el Índice Gingival, Índice de placa, Índice Hemorrágico en 10 sujetos para cada grupo. Se realizaron tres evaluaciones: una preliminar, la segunda a la semana de uso del enjuagatorio y la tercera a los 15 días.

Análisis descriptivo: EI análisis descriptivo demuestra una variación en el índice gingival de acuerdo al tiempo de exposición del tratamiento (Fig. 1) que evidencia una disminución en el grupo clorhexidina, algo se observa en el grupo manzanilla, aunque más evidente. El grupo placebo no posee cambios que es lo esperable.

Para el índice de placa de acuerdo al tiempo de exposición del tratamiento (Fig. 2) se observa una disminución en el grupo clorhexidina (gold estándar), similar disminución se encuentra en el grupo manzanilla. El grupo placebo no posee cambios.

Para el índice hemorrágico de acuerdo al tiempo de exposición del tratamiento (Fig. 3) que establece una disminución en el grupo clorhexidina (gold estándar), en el grupo manzanilla alcanza la misma variación. El grupo placebo no presenta cambios. 
Análisis inferencial: Se comparó el Índice Gingival en los 3 tiempos para cada tratamiento. En el caso del Grupo de Placebo no se observó cambio significativo entre los tres tiempos evaluados (valor $p=0,192)$ se utilizó Dócima de Friedman. En el caso del grupo sometido a Manzanilla y Clorhexidina, si se encontró diferencia significativa (valor $p \sim 0,000$, en ambos casos), en este caso se utilizó ANOVA de medidas repetidas.

Se comparó el Índice de placa en los 3 tiempos para cada tratamiento. En el caso de Placebo no se observó un cambio signficativo entre los tres tiempos evalaudos a través de la Docima de Friedman (valor $p=0,239$ ). En el caso del grupo de Manzanilla y Clorhexidina se encontró diferencia significativa el cual se empleó ANOVA de medidas repeticas (valor $p \sim 0,011$ y $p \sim 0,001$ ) .

Se comparó del Índice hemorrágico en los 3 tiempos para cada tratamiento. En el caso del Placebo no hubo diferencia significativa en el cual se utilizo la Dócima de Friedman (valor $p=0,607$ ). Para el caso del grupo de Manzanilla y Clorhexidina si hubo diferencia significativa a traves de loa Docima de Friedman (valor $p=0,01$ y valor $p \sim 0,00$ ).

Cuando se analizan los resultados de los grupos desde el inicio del tratamiento hasta la primera semana para el Índice Gingival en los tres tratamientos si existe diferencia significativa a la primera semana de uso, se utilizo ANOVA de medidas repetidas (valor $p=0,008$ ). Para el efecto de la primera a la segunda semana de uso del colutorio para el índice gingival en los dos tratamientos más el placebo hubo diferencia significativa con Kruskal-Wallis (valor $p$ $=0,04)$. Para el efecto desde el inicio de los tratamientos hasta la segunda semana si hubo diferencia significativa, se ocupo la Prueba de ANOVA de medidas repetidas (valor $p \sim 0,000$ ).

Para el Índice de Placa desde el inicio del tratamiento a la primera semana hubo diferencia significativa en los tres tratamientos, se utilizo ANOVA de medidas repetidas (valor $p=0,014$ ) Para el efecto de los tratamientos de la primera semana a la segunda semana no hubo diferencia significativa (valor $p=0,91)$. Para el efecto desde el inicio de los tratamientos hasta la segunda semana de uso si hubo diferencia significativa (valor $p=0,01$ ). Para el cual se utilizó la Prueba de Kruskal-Wallis.

Para la variable Índice Hemorrágico desde el inicio del tratamiento a la primera semana hubo diferencia significativa en los tres tratamientos se realizo a través de la Prueba de ANOVA de medidas repetidas (valor $p=0,08$ ). Desde el primer control al segundo control no hubo diferencia significativa (valor $p=0,17)$. Desde el inicio del tratamiento al segundo control no hubo diferencia significativa (valor $p=0,01)$. Para el cual se utilizó la Prueba de KruskalWallis.

Efectos adversos: no se observaron efectos adversos en los pacientes que utilizaron el colutorio de manzanilla en los tiempos evaluados.

\section{DISCUSION}

La utilización de colutorios en odontología es parte de las recomendaciones para mantener una buena higiene oral, sin embargo su efectividad es limitada por no producir efectos adversos. Enjuagues que compartan las características antimicrobianas de los colutorios con clorhexidina de carácter natural y que posean efectos beneficiosos por sobre los adversos. Por lo cual el ensayo clínico que compara la efectividad de los colutorios de manzanilla, clorhexidina y placebo como control demostró que existen diferencias significativas entre los dos primeros grupos, y el tercero se comportó de acuerdo a lo esperado (no hubo diferencia significativa en el tiempo en las tres variables). Al cabo de 2 semanas de aplicación del colutorio, los 3 tratamientos produjeron una disminución significativa del promedio

Resultados de estudios similares (Pourabbas et al.) utilizaron un colutorio de chamonilla alemana, en pacientes con gingivitis, utilizándola dos veces al día durante 4 semanas. Concluyeron que puede reducir tanto la acumulación de placa como la inflamación gingival. Otros investigadores (Albuquerque et al., 2010), evaluaron la actividad antiadherente del extracto de Matricaria recutita Linnesta en las bacterias del biofilm dental. Se observó que Matricaria demuestra un efecto en la inhibición de la síntesis de glucano representada por la inhibición de adhesión de estos microorganismos al biofilm.

En este estudio no se controló si al momento del examen se habían cepillado los dientes, ya que este factor influye directamente en el índice de placa. Se propone considerar este sesgo en un nuevo estudio. 

controlado. Int. J. Odontostomat., 6(2):151-156, 2012.

Se concluye que el Colutorio de extracto de Matricaria recutita I, tipo Manzanilla Primavera Puelche reduce la inflamación gingival.

GAETE, F. M. J. \& OLIVA, M. P. Effectiveness of chamomile mouthwash compared with placebo and chlorhexidine in patients with gingivitis aged 19 to 25 years: controlled clinical trial. Int. J. Odontostomat., 6(2):145-150, 2012.

ABSTRACT: Gingivitis is a periodontal disease of high prevalence in the population. Mechanical control of bacterial plaque and the use of a chemical agent help for the treatment and prevention. The aim of this research is to determine the effectiveness chamomille mouthwash compared with placebo and chlorhexidine $(0.12 \%)$ in the reduction of gingival inflammation in patients with gingivitis between 19 and 25 years old. Was perfomed a clinical trial, through non-probability sampling. Involved 30 subjects who were randomized into three groups assigned to each treatment: Chamomile, Clorhexidine $(0.12 \%)$ and Placebo. Was controlled at 7 and 15 days of use of the mouthwash. We evaluated gingival index, plaque index and bleeding index. Statistical analysis was conducted which measures the normality by Shapiro Wilk test, an Anova for repeated measures for evaluating partners. Data showed significant differences in the statistical analysis parametric an nonparametric. The Chamomile mouthwash is effective in reducing inflammation and Chlorhexidine compared with Placebo.

KEY WORDS: mouthwash, chamomile, chlorhexidine, gingival inflammation.

\section{REFERENCIAS BIBLIOGRÁFICAS}

Albuquerque, A.; Pereira, M.; Pereira, J.; Pereira, L.; Silva, D.; Macedo-Costa, M. \& Higino, J. Efeito antiaderente do extrato da Matricaria recutita Linn. sobre microorganismos do bioflme dental. Rev. Odontol. UNESP, 39(1):21-5, 2010.

Bernimoulin, J. P. Recent concepts in plaque formation. J. Clin. Periodontol., 30 (Supp/ 5):7-9, 2003.

Calsina-Gomis, G. \& Serrano-Granger, J. ¿Existen realmente diferencias clínicas entre las distintas concentraciones de clorhexidina?: Comparación de colutorios. RCOE, 10(4):457-64, 2005.

Cárcamo, V.; Gonzalez, P. \& Salgado, F. Frecuencia de uso clínico eficaz del colutorio de manzanilla, en funcionarios de la Facultad de Odontología de la Universidad del Desarrollo. Tesis de la Facultad de Odontología de la Universidad del Desarrollo, Santiago, Chile, 2008.

George, J.; Hedge, S.; Rajesh, K. S. \& Kumar, A. The efficacy of a herbal-based toothpaste in the control of plaque and gingivitis: A clinico-biochemical study. Indian J. Dent. Res., 20(4):480-2, 2009.

Mandel, I. D. Chemotherapeutic agents for controlling plaque and gingivitis. J. Clin. Periodontol., 15(8):488-98, 1988.

Mckay, D. L. \& Blumberg, J. B. A Review of the bioactivity and potential health benefits of chamomile tea (Matricaria recutita L.). Phytother. Res., 20(7):519-30, 2006.

Mullally, B. H; James, J. A.; Coulter, W. A. \& Linden, G. $J$. The efficacy of a herbal- based toothpaste on the control of plaque and gingivitis. J. Clin. Periodontol., 22(9):686-9, 1995.

Ozaki, F.; Pannuti, C. M.; Imbronito, A. V.; Pessotti, W.; Saraiva, L.; de Freitas, N. M.; Ferrari, G. \& Cabral, V. N. Efficacy of a herbal toothpaste on patients with established gingivitis a randomized controlled trial. Braz. Oral Res., 20(2):172-7, 2006.

Paixão, C. C. B.; Santos, A.; Oliveira, C.; Silva, L. \& Nunes, M. A. Uso de plantas medicinais en pacientes portadores de afecoces bucals. Odontol. Clín.Cient., 1(1):23-7, 2002.

Pereira, J.; Pererira, M.; Sampaio, F.; Correia, M.; Pollianna, M.; Ferreira, C. \& Higin, J. Efeito antibacteriano e antiaderente in vitro do extrato da Punica granatum Linn. sobre microrganismos do biofilme dental. Rev. Bras. Farmacogn., 16(1):8893, 2006.

Saxer, U. P.; Menghini, G.; Bohnert, K. J. \& Ley, F. El efecto de dos pastas de dientes en la placa y la inflamación gingival. J. Clin. Dent., 6(2):154-6, 1995.

Pourabbas, R.; Delzar, A. \& Chitsaz, M. The effect of 
GAETE, F. M. J. \& OLIVA, M. P. Efectividad del colutorio de manzanilla comparado con placebo y clorhexidina en pacientes con gingivitis entre 19 y 25 años: ensayo clínico controlado. Int. J. Odontostomat., 6(2):151-156, 2012.

German chamomile mouthwash on dental plaque and gingival inflammation. Iranian J. Pharm. Res., 2:105-9, 2005.

Sadr Lahijani, M.; Raoof Kateb, H. R.; Heady, R. \& Yazdani, D. The effect of German chamomile (Marticariarecutita L.) extract and tea tree (Melaleucaalternifolia L.) oil used as irrigants on removal of smear layer: a scanning electron microscopy study. Int. Endod. J., 39(3):190-5, 2006.

Srivastava, J. K.; Shankar, E. \& Gupta, S. Chamomile: A herbal medicine of the past with bright future. Mol. Med. Report, 3(6):895-901, 2010.

Torres, C. R. G.; Kubo, C. H.; Anido, A. \& Rodrigues, J. R. Antimicrobial agents and your potential of use in odontology. Pós-Grad. Rev. Fac. Odontol. São José dos Campos, 3:43-52, 2000.

Weiss, R. F. Herbal Medicine. Beaconsfield, UK, Beaconsfield Publishers Ltda., 1988. pp.22-8.
Dirección para correspondencia:

María Jesús Gaete Forno

Facultad de Odontología

Universidad del Desarrollo, sede Concepción

Barros Arana 1734, Concepción

CHILE

Fono: 041-2268544

Fax: 56-41-2268501

Email: ma.jesusgaete@gmail.com

Recibido : 16-03-2012

Aceptado: 14-05-2012 